\title{
Development and validation of an interview-administered FFQ for assessment of vitamin D and calcium intakes in Finnish women
}

\author{
Suvi T. Itkonen*, Maijaliisa Erkkola, Essi Skaffari, Pilvi Saaristo, Elisa M. Saarnio, Heli T. Viljakainen, \\ Merja U. M. Kärkkäinen and Christel J. E. Lamberg-Allardt \\ Department of Food and Environmental Sciences, Calcium Research Unit, Division of Nutrition, University of Helsinki, \\ PO Box 66, FIN-O0014 University of Helsinki, Helsinki, Finland
}

(Submitted 16 April 2015 - Final revision received 16 December 2015 - Accepted 18 December 2015 - First published online 9 February 2016)

\begin{abstract}
Increased vitamin D fortification of dairy products has increased the supply of vitamin D-containing products with different vitamin D contents on the market in Finland. The authors developed a ninety-eight-item FFQ with eight food groups and with a question on supplementation to assess dietary and supplemental vitamin D and $\mathrm{Ca}$ intakes in Finnish women $\left(60^{\circ} \mathrm{N}\right)$. The FFQ was validated in subgroups with different habitual vitamin D supplement use (0-57.5 $\mu \mathrm{g} / \mathrm{d}$ ) against the biomarker serum 25-hydroxyvitamin D (S-25(OH)D) and against 3-d food records (FR) ( $n$ 29-67). Median total vitamin D intake among participants was 9.4 (range 1.6-30.5) $\mu \mathrm{g} / \mathrm{d}$. Spearman's correlations for vitamin D and Ca ranged from $0.28(P 0 \cdot 146$, FFQ $v$. S-25(OH)D, persons not using supplements) to $0.75(P<0 \cdot 001$, FFQ $v$. FR, supplement use included). The correlations between the FFQ and S-25(OH)D concentrations improved within increasing supplement intake. The Bland-Altman analysis showed wide limits of agreement between FFQ and FR: for vitamin D between -7.8 and $8.8 \mu \mathrm{g} / \mathrm{d}$ and for Ca between -938 and $934 \mathrm{mg} / \mathrm{d}$, with mean differences being $0.5 \mu \mathrm{g} / \mathrm{d}$ and $2 \mathrm{mg} / \mathrm{d}$, respectively. The triads method was used to calculate the validity coefficients of the FFQ for vitamin $\mathrm{D}$, resulting in a mean of $1.00(95 \% \mathrm{CI} 0.59,1.00)$ and a range from 0.33 to 1.00 . The perceived variation in the estimates could have been avoided with a longer FR period and larger number of participants. The results are comparable with earlier studies, and the FFQ provides a reasonable estimation of vitamin D and Ca intakes.
\end{abstract}

\section{Key words: Vitamin D: Calcium: FFQ: Validity: Reproducibility: Method of triads}

Adequate vitamin D intake is important for bone health, and low vitamin D status may increase the risk of many common chronic diseases. In northern latitudes, exposure to UVB light is limited, and the formation of vitamin D in the skin occurs only during March-October ${ }^{(1)}$. This causes large variation in serum 25-hydroxyvitamin D (S-25(OH)D) status between the seasons; vitamin D status during winter in Finland has been low ${ }^{(2)}$. A nutritional policy intervention to this was vitamin D fortification of fluid dairy products and spreads, which has increased vitamin D intake in Finland in recent years ${ }^{(3)}$. Today, these comprise the main dietary vitamin D sources, other important sources being fish and fish products ${ }^{(4)}$. The use of vitamin D supplements has also increased: in the National FINDIET 2012 Study $56 \%$ of the females and $34 \%$ of the males used vitamin D supplements ${ }^{(4)}$.

Assessing vitamin D intake with food records (FR) or recalls entails some problems - for example, rich vitamin D sources consumed rarely, such as fish products, may not be included in the short recording periods. FR and recalls are also burdensome and expensive to carry out ${ }^{(5)}$. Thus, a need exists for a trustworthy and low-cost method to assess vitamin D intake: a FFQ.
As $\mathrm{Ca}$ is also present in many foodstuffs containing vitamin $\mathrm{D}$, it is easy to measure $\mathrm{Ca}$ intake together with vitamin $\mathrm{D}$ intake; both are important nutrients when investigating, for example, bone health.

Many studies have validated FFQ concerning vitamin D and $\mathrm{Ca}$, but validation against a biomarker has not been studied extensively ${ }^{(6-10)}$. In the present study, we used the biomarker S-25(OH)D and FR as a gold standard against which the FFQ was validated. The aim of this study was to develop and validate a reliable tool to assess dietary and supplemental vitamin D and $\mathrm{Ca}$ intakes in a Finnish population.

\section{Methods}

\section{Participants}

The study was carried out as a substudy of the intervention study BreaD, which was conducted as a part of the European Union-funded research project ODIN (Food-based Solutions For Optimal Vitamin D Nutrition And Health Through The Life Cycle) (FP7-613977-ODIN). The study was carried out at the

Abbreviations: FR, food records; S-25(OH)D, serum 25-hydroxyvitamin D

* Corresponding author: S. T. Itkonen, email suvi.itkonen@helsinki.fi 
Viikki Campus of the University of Helsinki $\left(60^{\circ} \mathrm{N}\right)$ in Finland in January-April 2014, when there is not enough UVB irradiation exposure for endogenous vitamin $\mathrm{D}$ production and when S-25 $(\mathrm{OH}) \mathrm{D}$ concentrations are expected to be at the lowest ${ }^{(1)}$. The voluntary participants were mainly recruited from the University Campus areas by advertisements for personnel, through student mailing lists and through Internet by social media advertisements. This study was conducted according to the guidelines laid down in the Declaration of Helsinki, and all procedures involving human subjects were approved by the Helsinki Uusimaa Hospital District Ethics Committees. Written informed consent was obtained from all the subjects.

Inclusion criteria for the BreaD study were female sex, good general health (no continuous medication), Caucasian origin, BMI $<30 \mathrm{~kg} / \mathrm{m}^{2}$ and age between 20 and 40 years. The final exclusion criteria were diagnosed coeliac disease or cereal allergy, a vacation in a sunny place during the study or past 2 months, regular use of vitamin D supplements $>15 \mu \mathrm{g} / \mathrm{d}$ and pregnancy or breast-feeding during the study. A total of fiftyfour participants, who qualified for the participation, were screened pre-experimentally for S-25(OH)D concentration. Of them, forty-one participants with the lowest S-25(OH)D concentrations were allowed to participate in the BreaD study. All the participants of the BreaD study automatically participated in the validation study. In addition, subjects excluded from the BreaD Study ( $n$ 13) were asked to participate in the present validation study and nine of them agreed to participate (with maximum regular daily vitamin $\mathrm{D}$ supplement dose among those participants being $25 \mu \mathrm{g} / \mathrm{d}$ ). However, five participants discontinued participation, thus only one FFQ and one FR were available from them (in the reproducibility study, $n$ 45). In addition, seventeen students were recruited for the validation study, for the interview for the FFQ and for S-25(OH)D blood samples collection without limitation on supplement use and sunny holidays to widen the range of vitamin $\mathrm{D}$ intake, thus adding advantage for the validation against S-25(OH)D.

\section{Development of the FFQ}

The purpose of the FFQ was to collect data on habitual vitamin D and $\mathrm{Ca}$ intakes. The FFQ was adapted from an existing fiftytwo-item questionnaire used in several studies by our research group in Finland ${ }^{(11,12)}$. Increased vitamin $\mathrm{D}$ fortification of dairy products has widely increased the supply of vitamin D-containing products with different vitamin D contents on the market. In our study, data on the use and supply of available vitamin D- and Ca-fortified products were examined to update the FFQ. Foods considered as important sources of vitamin D and $\mathrm{Ca}$, based on nutrient content and consumption frequency, were included in the FFQ. Finally, a ninety-eight-item FFQ divided into eight food groups was developed (Table 1). The FFQ also included detailed questions on the use of supplements (frequency of use, brand name, dose). The intake calculations of vitamin $\mathrm{D}$ and $\mathrm{Ca}$ were based on food consumption frequencies during the previous month. The following alternatives for frequencies were included: portions/d, portions/ week, portions/month, rarer or non-consumption. The questions of frequency and portion size were closed. The mean portion
Table 1. Food groups and examples of products in the FFQ

\begin{tabular}{|c|c|}
\hline Food groups & Examples of products \\
\hline $\begin{array}{l}\text { Dairy products and milk-based } \\
\text { soups }\end{array}$ & $\begin{array}{l}\text { Vitamin D-fortified milk/sour milk/ } \\
\text { yoghurt } \\
\text { Salmon soup, milk based }\end{array}$ \\
\hline Cereals & Porridge, made with milk; pizza* \\
\hline Main dishes, meat dishes & Liver sausage; pasta casserole* \\
\hline Fish and eggs & $\begin{array}{l}n-3 \text { and vitamin D-fortified egg } \\
\text { Roe; salmon; baltic herring; pike- } \\
\text { perch }\end{array}$ \\
\hline $\begin{array}{l}\text { Fruits, berries, vegetables, } \\
\text { mushrooms }\end{array}$ & $\begin{array}{l}\text { Vitamin D-fortified special juice } \\
\text { Chanterelle, bolete }\end{array}$ \\
\hline Nuts, chocolate & Almonds $^{*}$, milk chocolate ${ }^{*}$ \\
\hline $\begin{array}{l}\text { Meal replacements/recovery } \\
\text { beverages }\end{array}$ & Special trademarks \\
\hline Mineral waters/vitamin drinks & Special trademarks \\
\hline
\end{tabular}

* Only for Ca intake.

sizes were adapted from the Finnish portion-size guide ${ }^{(13)}$. Open questions were asked concerning, for instance, the consumption, type and amount of fat spread on bread, the consumption of fat spread in porridge, the consumption of home-baked sweet buns (baked with fat spread) and the consumption of particular vitamin D-fortified cereals, muesli or snack bars. The type of fat used for cooking (as well the frequency of cooking at home) was asked in order to identify potential vitamin $\mathrm{D}$ sources in cooking.

The administration of the FFQ was carried out by an interviewer. A picture booklet of vitamin D-fortified products was used to aid the participants in identifying consumed products. The questionnaire was pre-tested in a small population ( $n$ 10) before administration in the validation study. The $\mathrm{Ca}$ and vitamin D content of the foodstuffs was obtained from the Finnish food composition database Fineli ${ }^{\circledR}$, which is developed and continuously updated by the Finnish National Institution of Health and Welfare (www.fineli.fi). The average duration of a FFQ interview was 20-25 min, ranging from 15 to $40 \mathrm{~min}$.

\section{The collection of food intake, background and biomarker data}

Habitual dietary intake data of the participants were collected using 3-d FR completed on consecutive days, which were randomised to collect balanced data on weekdays and weekends. The participants were instructed to maintain their normal food habits during the recording period and to record all foods and beverages immediately after consumption. Nutrient intake was calculated using a computer-based programme (AivoDiet) based on Fineli ${ }^{\circledR}$. The supplemental vitamin D intake was collected as in the FFQ.

After 12-h of fasting, blood samples were collected, and participants' height and weight were measured. Subjects were advised to fill in the study form, which included a background questionnaire, which also contained questions on possible sunlight exposure. All forms were checked by the researchers, and additional information was requested if needed. Fasting S-25(OH)D was measured as a marker of vitamin D status with OCTEIA immunoenzymometric assay (IDS). Inter- and intra-assay $\mathrm{CV} \%$ were $2 \cdot 7$ and $3 \cdot 2$, respectively, based on the 
provided controls measured in the laboratory. Reproducibility was ensured by adhering to the Vitamin D External Quality Assessment Scheme, DEQAS (deqas.kpmd.co.uk/). The laboratory received the DEQAS proficiency certificate in 2012. The mean bias of DEQAS samples analysed by our method was $6.6 \%$ in relation to the DEQAS mean and $7.5 \%$ in relation to the DEQAS target value (results from October 2013 to January 2014).

\section{Statistical analysis}

Statistical analysis was performed using SPSS version 22 (IBM). Vitamin D intake was calculated separately from diet and supplements, and $\mathrm{Ca}$ intake was measured only from the diet. The normality and homogeneity of the data were verified by the Kolmogorov-Smirnov test. S-25(OH)D and vitamin D intakes were not normally distributed, thus medians and ranges are presented. Ca intake was normally distributed, and the data are presented as mean values and standard deviations. In addition, interquartile ranges are presented. Comparisons between the groups were carried out by non-parametric tests (related-samples Wilcoxon's signed rank test) for vitamin $D$ variables and by paired-samples $t$ tests for Ca variables. $P$ values $<0.05$ were considered to be significant. The analyses were also carried out by excluding those participants from the student group who had been on sunny holidays during the last few months ( $n$ 6), with no impact on results. The bootstrapping to calculate $95 \%$ CI for the validity coefficients for the method of triads was performed by R programme version 3.0.2 (The R Foundation for Statistical Computing).

We analysed the data in the following manner - all participants ( $n$ 67) with unrestricted supplement use (blood samples and FFQ available), subgroup 1 with restricted supplement use $(0-25 \mu \mathrm{g} / \mathrm{d}, n 50$, blood samples, FFQ and FR available) and subgroup 2 who did not use supplements ( $n$ 29, blood samples, FFQ and FR available) - to perceive the possible differences in the results of validation within different supplemental vitamin D intakes. Spearman's rank correlation coefficients were used to assess the correlation between intake measured by the FFQ (vitamin D and Ca), FR (vitamin D and Ca) and S-25(OH)D to calculate the validity coefficients for the method of triads (see below). Cross-classification into quartiles of intake by FFQ (vitamin D and Ca), FR (vitamin D and Ca) and S-25(OH)D was used to compare the classification of one method in relation to another. The weighted $\kappa$ coefficients with $95 \%$ CI for the quartiles were also calculated ${ }^{(14)}$. The $\kappa$ values were interpreted as follows: $<0$, no agreement with the methods; 0-0.20, slight agreement; $0 \cdot 21-0.40$, fair agreement; 0.41-0.60, moderate agreement; 0.61-0.80, substantial agreement; and 0.81-1.00, almost perfect agreement ${ }^{(14)}$. Bland-Altman plots with $95 \%$ limits of agreement were calculated to visualise the mean difference of agreement between the FFQ and FR for vitamin D intake and $\mathrm{Ca}$ intake from food ${ }^{(15)}$.

The method of triads is applied in validation studies of dietary intake to evaluate the correlation between three measurements (FFQ, reference method and biomarker) and the theoretical true intake using validity coefficients $(\rho)^{(16)}$. We used the method of triads to determine the validity coefficients in the subgroup with restricted supplement use that had all three measurements available. With this method, the errors between the dietary assessment methods differ from the ones of the biomarker; thus, more accurate validity estimates can be provided. The correlations between the three methods ( $\mathrm{S}-25(\mathrm{OH}) \mathrm{D}, \mathrm{FR}, \mathrm{FFQ})$ were used to calculate validity coefficients by the method of triads ${ }^{(16)}$ and interpreted as upper limits of the validity coefficients, whereas the correlation between the dietary method and the biomarker were used as the lower limit of the validity coefficient. The following equation was used to calculate the validity coefficient for the FFQ:

$$
\rho \mathrm{QI}=\sqrt{\frac{r \mathrm{QR} \times r \mathrm{QB}}{r \mathrm{BR}}},
$$

where $\rho$ QI represents the validity coefficient between FFQ and true intake, $r \mathrm{QR}$ the correlation between FFQ and FR, $r \mathrm{QB}$ the correlation between FFQ and biomarker and $r$ BR the correlation between biomarker and FR. The method has been described in detail in the paper by Yokota et al. ${ }^{(16)}$. The $95 \%$ CI for the validity coefficients were calculated by bootstrapping 1000 samples of equal size ( $n$ 50) with replacement from the study participants.

Reproducibility of the FFQ for dietary intake was assessed by comparing means and assessing Spearman's correlations between the 1st and the 2nd FFQ, with an 8-week interval ( $n$ 45) (vitamin D and Ca). Cross-classification into quartiles of intake by the 1st and 2nd FFQ (dietary vitamin D and dietary Ca) was used to compare the classification of one method in relation to another. The weighted $\kappa$ coefficients with $95 \% \mathrm{CI}$ for the quartiles were also calculated ${ }^{(14)}$.

\section{Results}

\section{Characteristics of the participants}

All participants were females, aged between 20 and 37 years (mean 27 years), with mean BMI of 22.4 (range 18.3-29.1) $\mathrm{kg} / \mathrm{m}^{2}$. The mean height of the participants was 165.6 (range 154.0-175.5) cm, and the mean weight was 61.5 (range $44 \cdot 3-83 \cdot 2) \mathrm{kg}$. The S-25(OH)D and vitamin D and Ca intake characteristics of the participants in different subgroups are presented in Table 2. The median S-25(OH)D concentration was almost similar to the mean of the general population in Finland (all participants $63.6 \mathrm{nmol} / \mathrm{l}$; in Health 2011 Survey approximately $70 \mathrm{nmol} / \mathrm{l}^{(17)}$. Compared with the national recommendation of vitamin D intake in Finland $(10 \mu \mathrm{g} / \mathrm{d})^{(18)}$, the median total vitamin D intake was sufficient $(14.8 \mu \mathrm{g} / \mathrm{d})$, except in the subgroup not taking vitamin D supplements $(9.1 \mu \mathrm{g} / \mathrm{d})$; however, intake from food was similar between all participants and subgroups (Table 2). The median vitamin D intake from supplements among supplements users was $10 \mu \mathrm{g} / \mathrm{d}$. The average Ca intake among study participants was high (about $1250 \mathrm{mg} / \mathrm{d}$ ). It was, however, similar to that in the general population ${ }^{(4)}$ and above the recommended intake $(800 \mathrm{mg} / \mathrm{d})^{(18)}$. The S-25(OH)D concentrations and total vitamin $\mathrm{D}$ intake decreased in the subgroups according to lowering amounts of supplemental intake. 
Table 2. Serum 25-hydroxyvitamin $D(\mathrm{~S}-25(\mathrm{OH}) \mathrm{D}$ ) concentrations and intakes of vitamin $\mathrm{D}$ (from food, supplements and total) and Ca (food) assessed by FFQ and food records (FR) stratified by different supplement intakes among studied Finnish Caucasian origin women (Medians and ranges, interquartile range (IQR) with cut-offs 25 and $75 \%$; mean values, standard deviations and IQR)

\begin{tabular}{|c|c|c|c|c|c|c|}
\hline & \multirow{2}{*}{\multicolumn{2}{|c|}{$\begin{array}{l}\text { Unrestricted supplement use } \\
\qquad \text { (n } 67 \text { (54\% users)) }\end{array}$}} & \multicolumn{2}{|r|}{ Subgroup 1} & \multicolumn{2}{|r|}{ Subgroup 2} \\
\hline & & & \multicolumn{2}{|c|}{$\begin{array}{l}\text { Restricted supplement use } \\
(0-25 \mu \mathrm{g} / \mathrm{d})(\text { n } 50 \text { (42\% users) })\end{array}$} & \multicolumn{2}{|c|}{$\begin{array}{l}\text { No supplements }(0 \mu \mathrm{g} / \mathrm{d}) \\
(n 29)\end{array}$} \\
\hline & Medians & Ranges; IQR & Medians & Ranges; IQR & Medians & Ranges; IQR \\
\hline \multirow{2}{*}{\multicolumn{7}{|c|}{ Vitamin D intake from food $(\mu \mathrm{g} / \mathrm{d})^{\star}$}} \\
\hline & & & & & & \\
\hline FFQ & $9 \cdot 4$ & $1 \cdot 6-30 \cdot 5 ; 6 \cdot 2-12 \cdot 4$ & $9 \cdot 2$ & $1 \cdot 6-30 \cdot 5 ; 5 \cdot 9-11 \cdot 4$ & 9.1 & $1 \cdot 6-16 \cdot 7 ; 6 \cdot 0-11 \cdot 4$ \\
\hline FR & & N/A & $7 \cdot 6$ & $2 \cdot 5-26 \cdot 0 ; 5 \cdot 1-10 \cdot 4$ & $7 \cdot 7$ & $2 \cdot 7-26 \cdot 0 ; 6 \cdot 0-10 \cdot 5$ \\
\hline Vitamin D intake from supplements $(\mu \mathrm{g} / \mathrm{d})$ & $3 \cdot 2$ & $0-57.5 ; 0-10.0$ & 0 & $0-25 ; 0-8 \cdot 6$ & & 0 \\
\hline Median intake among the users $(\mu \mathrm{g} / \mathrm{d})$ & & $10 \cdot 0$ & & $10 \cdot 0$ & & 0 \\
\hline \multicolumn{7}{|c|}{ Total vitamin D intake (food + supplements) $(\mu \mathrm{g} / \mathrm{d})^{\star}$} \\
\hline FFQ & 14.8 & $1 \cdot 6-64 \cdot 3 ; 9 \cdot 2-23.5$ & 11.4 & $1 \cdot 6-40.5 ; 8.0-18 \cdot 4$ & $9 \cdot 1$ & $1 \cdot 6-16 \cdot 7 ; 6 \cdot 0-11.4$ \\
\hline FR & & $\mathrm{N} / \mathrm{A}$ & $11 \cdot 2$ & $2 \cdot 7-34 \cdot 7 ; 6 \cdot 7-18 \cdot 2$ & $7 \cdot 7$ & $2 \cdot 7-26 \cdot 0 ; 6 \cdot 0-10 \cdot 5$ \\
\hline \multicolumn{7}{|l|}{$\mathrm{Ca}$ intake from food $(\mathrm{mg} / \mathrm{d}) \dagger$} \\
\hline \multicolumn{7}{|l|}{ FFQ } \\
\hline Mean & & 1284 & & 1248 & & 1251 \\
\hline $\mathrm{SD} ; \mathrm{IQR}$ & & 542; 927-1546 & & 586; 829-1553 & & $648 ; 697-1560$ \\
\hline \multicolumn{7}{|l|}{ FR } \\
\hline Mean & & $N / A$ & & 1250 & & 1214 \\
\hline $\mathrm{SD} ; \mathrm{IQR}$ & & $\mathrm{N} / \mathrm{A}$ & & $461 ; 926-1582$ & & $501 ; 865-1561$ \\
\hline
\end{tabular}

* Related samples Wilcoxon's signed rank test between FFQ and FR $P>0.05$.

$\dagger$ Paired-samples $t$ test between FFQ and FR $P>0.05$.

Table 3. Validity and reproducibility of the FFQ*

\begin{tabular}{|c|c|c|c|c|c|c|c|}
\hline & \multicolumn{3}{|c|}{ Cross-classification in quartiles } & \multicolumn{2}{|c|}{ Spearman's correlations } & \multicolumn{2}{|c|}{ Weighted $k$} \\
\hline & Same $(\%)$ & Same or adjacent $(\%)$ & Opposite (\%) & $r$ & $P$ & $\kappa$ & $95 \% \mathrm{Cl}$ \\
\hline \multicolumn{8}{|l|}{ Validity of the FFQ } \\
\hline \multicolumn{8}{|l|}{ Unrestricted supplement use $(0-57.5 \mu \mathrm{g} / \mathrm{d})(n 67)$} \\
\hline \multirow{2}{*}{\multicolumn{8}{|c|}{ Restricted supplement use $(0-25 \mu \mathrm{g} / \mathrm{d})(n 50)$}} \\
\hline & & & & & & & \\
\hline FFQ v. S-25(OH)D total vitamin D intake & 30 & 78 & 4 & 0.372 & 0.008 & -0.016 & $-0.126,0.094$ \\
\hline FFQ $v$. food record total vitamin D intake & 48 & 92 & 0 & 0.751 & $<0.001$ & 0.331 & $0.215,0.447$ \\
\hline FFQ $v$. food record vitamin $D$ intake from food & 40 & 80 & 2 & 0.559 & $<0.001$ & 0.169 & $0.053,0.285$ \\
\hline \multicolumn{8}{|l|}{ No supplement use ( $n$ 29) } \\
\hline $\begin{array}{l}\text { FFQ v. S- } 25(\mathrm{OH}) \mathrm{D} \text { vitamin } \mathrm{D} \text { intake from food } \\
\text { Ca intake from food }(n 50)\end{array}$ & 41 & 79 & 10 & 0.277 & $0 \cdot 146$ & 0.235 & $0.086,0.384$ \\
\hline FFQ $v$. food record & 32 & 92 & 0 & 0.696 & $<0.001$ & 0.011 & $-0.105,0.127$ \\
\hline \multicolumn{8}{|l|}{ Reproducibility of the FFQ } \\
\hline FFQ1 v. FFQ 2 vitamin D intake from food $\dagger$ & 56 & 82 & 0 & 0.694 & $<0.001$ & 0.395 & $0.270,0.517$ \\
\hline FFQ1 v. FFQ2 Ca intake from food $\dagger$ & 58 & 91 & 0 & 0.755 & $<0.001$ & 0.372 & $0.245,0.499$ \\
\hline
\end{tabular}

S-25(OH)D, serum 25-hydroxyvitamin D.

* Cross-classification comparison, Spearman's correlations and weighted $k$ values between FFQ $v$. other methods stratified by different supplement intakes.

† 8-week interval.

\section{Validity of the FFQ}

Vitamin D. Spearman's correlations between the FFQ and other methods in all comparisons were significant, ranging in subgroups from 0.56 to $0.75(P<0.001)$, except between the FFQ and S-25(OH)D in participants not using supplements (Table 3), where the correlation was non-significant $(r 0.28$, $P$ 0.146). The correlations between the FFQ and S-25(OH)D considerably weakened with decreasing supplemental intake, showing the strong effect of supplementation on S-25(OH)D. There was no major misclassification of participants into intake quartiles; most of them were classified into the same or adjacent quartiles (Table 3). Most misclassifications occurred in the quartile comparison between vitamin D intake from food (FFQ) and S-25(OH)D (10\%) among participants not using supplements. Limits of agreement for the FFQ and the FR for vitamin D intake from food are shown in Bland-Altman plots (Fig. 1(a)). The mean difference was $0.51 \mu \mathrm{g} / \mathrm{d}$ between the FFQ and FR, and the $95 \%$ limits of agreement for FFQ and FR for vitamin D intake were between -7.82 and $+8.83 \mu \mathrm{g} / \mathrm{d}$, indicating overestimation of vitamin $\mathrm{D}$ intake by the FFQ. The weighted $\kappa$ values in the restricted supplement use group ranged from poor to fair (Table 3), were slight in the unrestricted supplement use group and were fair among participants not using supplements (Table 3). 

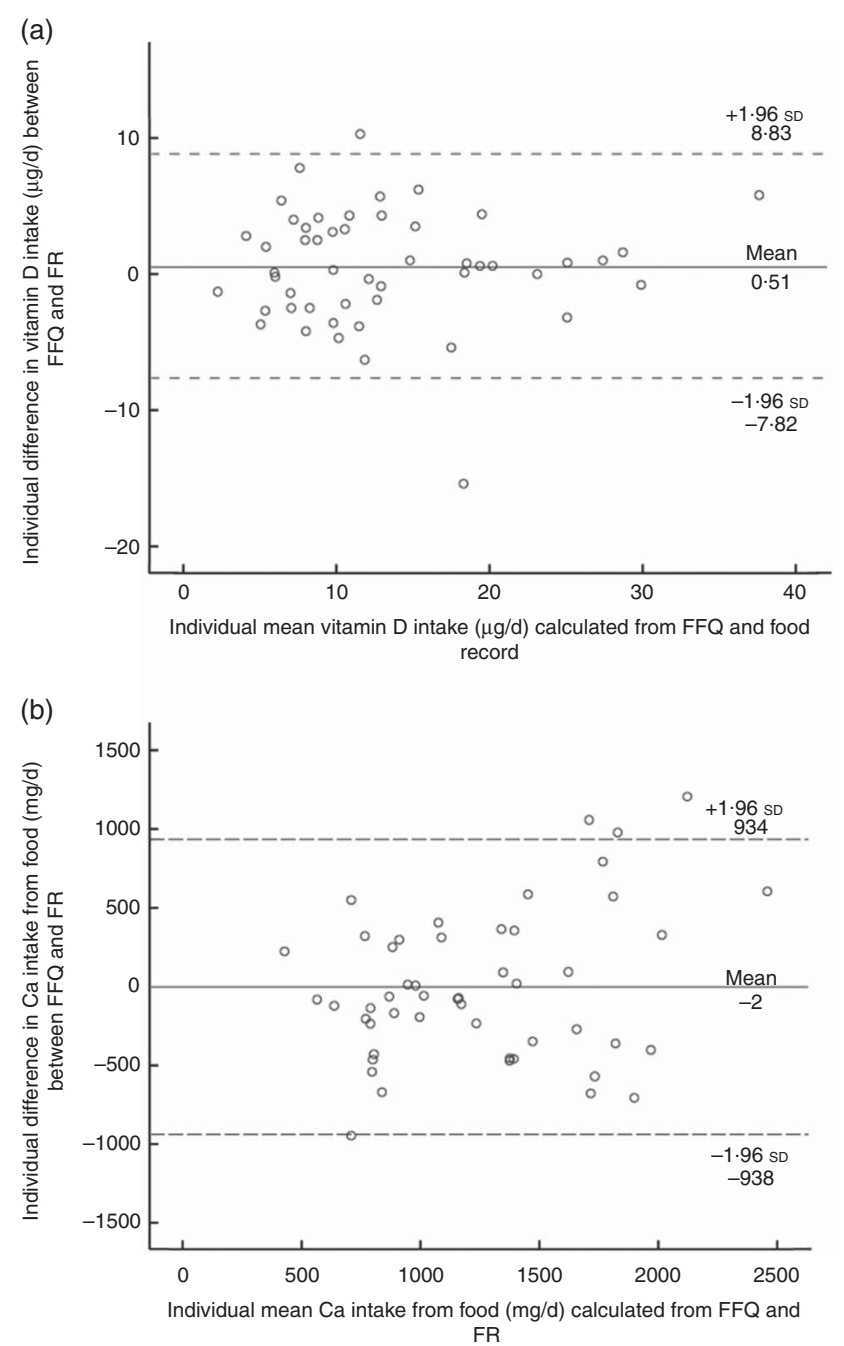

Fig. 1. Bland-Altman plots showing the mean difference (-) and $95 \%$ limits of agreement (------) between the FFQ and the food record (FR) for (a) vitamin $\mathrm{D}$ intake from food $(n 50)$ and (b) calcium intake from food $(n 50)$.

The method of triads was used in the subgroup with restricted supplement use ( $n 50)$ with all three vitamin D variables (FFQ, FR, S-25(OH)D) (see illustration, $\rho$ values, 95\% CI and ranges in (Fig. 2). The validity coefficient values $>1.00$ were observed and truncated to 1.00 . The method showed the highest validity coefficients between FFQ and the true intake and the lowest between the biomarker and the true intake (Fig. 1). In the subpopulation not taking supplements ( $n$ 29), the correlation between FR and S-25(OH)D was negative, which did not allow bootstrapping of the data ${ }^{(16)}$, and thus the method of triads could not be used in this subgroup analysis.

Calcium. Spearman's correlation between the FFQ and the FR was significant $(r 0 \cdot 70, P<0.001)$. In cross-classifications of $\mathrm{Ca}$ intake by both methods, none was classified into the opposite quartile (Table 3). However, 32\% of the participants were classified into the same quartile and $92 \%$ into the same or adjacent quartile. In the Bland-Altman plot (Fig. 1(b)), both

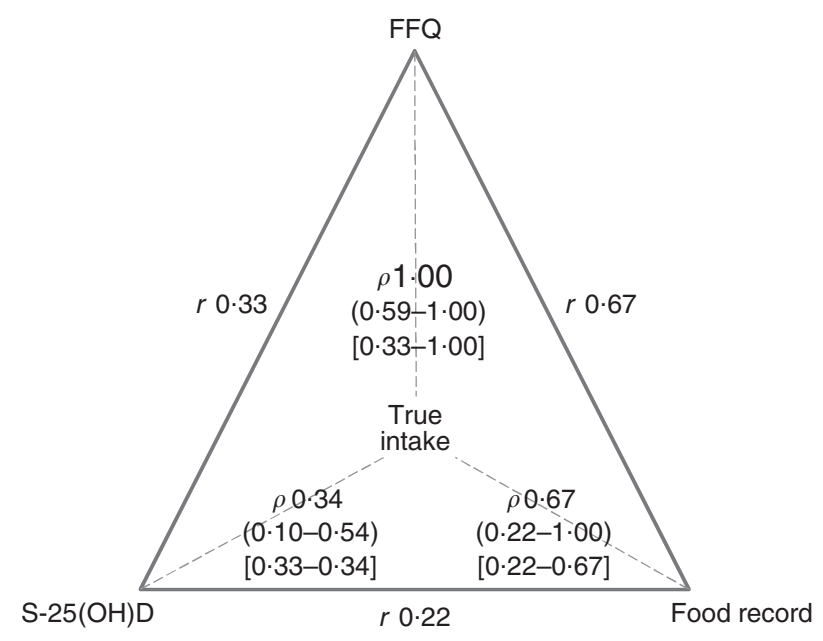

Fig. 2. Method of triads ( $n 50$ ): triangular comparison between the FFQ, food record and serum 25 -hydroxyvitamin $\mathrm{D}(\mathrm{S}-25(\mathrm{OH}) \mathrm{D})$. The correlations between FFQ and S-25(OH)D were used as the lower limit of the validity coefficients, and upper limits were calculated by the method of triads. All values $>1.00$ were truncated as this is the highest possible value. See calculation formulae in Yokota et al. ${ }^{(16)}$. $r$, Correlations between the methods; $\rho$, validity coefficient between each method and the true intake (in parentheses $(95 \% \mathrm{Cl}$ ) and [ranges]).

underestimations and overestimations of Ca intake measured by FFQ could be observed; the deviation tended to be higher with high $\mathrm{Ca}$ intake. The mean difference between the FFQ and the FR was $2 \mathrm{mg} / \mathrm{d}$, and the $95 \%$ limits of agreement for the $\mathrm{Ca}$ intake were between -938 and $+934 \mathrm{mg} / \mathrm{d}$. The weighted $\kappa$ value for the FFQ was not statistically significant.

\section{Reproducibility of the FFQ}

Results for the reproducibility study are shown in Table 3. Reproducibility of the FFQ for dietary intake (8-week interval) was good; correlation coefficients between the 1st and the 2 nd FFQ were for vitamin D $0.70(P<0.001)$ and for Ca 0.76 $(P<0 \cdot 001)$. In non-parametric comparison tests for vitamin $\mathrm{D}$ intakes, no significant differences between the 1 st and the 2nd FFQ were present (median vitamin D intake 9.2 (range $1.6-30 \cdot 5) \quad v .8 .5$ (range 3.5-21.0) $\mu \mathrm{g} / \mathrm{d}, P>0.05$ ). In pairedsamples $t$ test, no differences were seen in $\mathrm{Ca}$ intake between the 1 st and the 2nd FFQ (mean Ca intake 1248 (SD 481) v. 1242 (sD 586) $\mathrm{mg} / \mathrm{d}, P>0.05$ ). The weighted $\kappa$ values for reproducibility of the FFQ were fair for both vitamin D and Ca (Table 3). Cross-classification analysis showed no major misclassification $0 \%$ were classified into the opposite quartile for both vitamin D and Ca. Of vitamin D values, $82 \%$ were classified into same or adjacent quartile, as were $91 \%$ of the Ca values.

\section{Discussion}

We developed a ninety-eight-item FFQ to collect data on habitual vitamin D and $\mathrm{Ca}$ intakes in the Finnish population. This is the first study using the method of triads to assess the validity of a FFQ in measuring vitamin D intake carried out in an area with wide nutrition policy-based food fortification and 
considerably high supplemental consumption. Accordingly, validity of the FFQ for vitamin D and Ca was also assessed by cross-classification, weighted $\kappa$ analysis and Bland-Altman plots. The differences between the intake estimates calculated from FFQ and FR were considerably large when assessing vitamin $\mathrm{D}$ intake, indicating wider individual variation in the estimations of vitamin D intake than Ca intake. However, FFQ usually tend to overestimate intakes compared with $\mathrm{FR}^{(19)}$. Cross-classification analysis showed no major misclassification, and for both vitamin D and Ca most participants were classified into same or adjacent quartile. The $\kappa$ analysis mainly showed slightly fair agreement with wide 95\% CI. The evaluated methods correlated well but some variation in the estimates was observed. It is important to note that the correlations between FFQ and S-25(OH)D varied between the subgroups with different supplemental intakes, and the strongest correlations were found with the highest supplement intake. This shows the considerably strong effect of supplementation on S-25(OH)D and proves the importance of validating the FFQ within different supplementation levels.

The method of triads including biomarkers and the reference methods has been used to validate FFQ - for example, protein, $\beta$ carotene, vitamin $\mathrm{C}$ and cholesterol intakes in the European Prospective Investigation into Cancer and Nutrition (EPIC) Study ${ }^{(20)}$ and for carotenoid, vitamin E and PUFA intakes ${ }^{(21,22)}$. A recent Serbian study validated a vitamin D intake FFQ against plasma 25-hydroxyvitamin D (25(OH)D) and 24-h food recalls ${ }^{(10)}$. The method of triads assumes that the three measurements are linearly correlated, but the errors between the methods do not correlate ${ }^{(15)}$. In our study, the calculated validity coefficient theoretically showed strong correlation between the true intake and the intake assessed by the FFQ. However, the $95 \%$ CI were wide. The Heywood case $(\rho>1 \cdot 00)$ also occurred $^{(16)}$, indicating correlating errors between two or more assessment methods. This could be due to the nutrient contents of the foodstuffs originating from the same food composition database used in the FFQ and $\mathrm{FR}^{(5,22)}$, and may cause overestimation of the validity coefficients. Thus, the results of the triads should be interpreted with caution. Further, the negative correlations between FR and S-25(OH)D among the participants not using supplements ( $n$ 29) did not allow the sample to be bootstrapped and further analysed with the method. This indicates that a 3-d FR is not long enough to collect all dietary vitamin D sources from the present diet, whereas S-25(OH)D reflects the vitamin D intake from a longer time. To avoid negative correlations, Yokota et al. $^{(16)}$ recommend a sample size of at least fifty subjects. The phenomenon has also been observed in earlier studies. McNaughton et al. ${ }^{(21)}$ were not able to validate the data with the method of triads for vitamin $\mathrm{E}$ and $\beta$ cryptoxanthin due to the negative correlations, which was also reflected by the small sample size $(n<30)$. However, our study is one of the first attempts to use the triads method for validating a FFQ for vitamin $\mathrm{D}$, and further research with larger populations is needed. As Yokota et al. ${ }^{(16)}$ state, the use of the method of triads does not exclude the need for Bland-Altman analysis in the validation studies. In our study, Bland-Altman analysis showed overestimation of vitamin D intake from food by the FFQ with wide limits of agreement. The
FFQ also showed both underestimations and overestimations of $\mathrm{Ca}$ intake compared with the FR. However, for the Bland-Altman method, the recommended smallest sample size is 50 , which we had in the comparison of the FFQ with the $\mathrm{FR}^{(5)}$. The weighted $\kappa$ statistics showed different levels of agreement within the supplementation groups. In the study of Masson et $a l^{(23)}$, the weighted $\kappa$ values for the agreement between a self-administered FFQ and 4-d weighted FR were fair for vitamin D intake and moderate for Ca intake. Misclassification into the opposite third was present in $10-13 \%$ of vitamin D values, as were $5-7 \%$ of the Ca values. In our study, the $\kappa$ values showed poor-to-fair agreement, which is probably explained by smaller subgroups. Misclassification rates were, however, smaller compared with the report of Masson et al. ${ }^{(23)}$.

The validation of vitamin $\mathrm{D}$ intake measured by a FFQ against a biomarker (here, S-25(OH)D) is rare. In the systematic review of Henríquez-Sánchez et $a l^{(6)}$ (studies conducted before March 2008), only one study of the twenty-one included had used a biomarker as a reference method to assess the validity of the FFQ. FR were used in fourteen of these studies. Jacques et $a l^{(24)}$ compared the vitamin $\mathrm{D}$ intake derived from an interview-driven FFQ and plasma $25(\mathrm{OH}) \mathrm{D}$, finding a correlation of 0.35 when supplements were included and 0.25 when they were not. However, the correlations are generally better when specific questions about supplement use are included ${ }^{(6)}$, as in our FFQ. In earlier validation studies on vitamin intakes, only $27 \%$ used a biomarker as a reference method, and the correlation coefficients varied between 0.26 and $0.38^{(6)}$, which is considerably less than that in our study $(r$ 0.37-0.55), when the supplement use was included, but similar to our results without supplements $(r$ 0.28). A recent study validated a self-administered vitamin D FFQ against 24-h food recalls and plasma $25(\mathrm{OH}) \mathrm{D}$ by the method of triads in Serbia where no vitamin $\mathrm{D}$ fortification is used ${ }^{(10)}$. The validity coefficients were better than ours, probably because the range of vitamin $\mathrm{D}$ intake was very narrow and the vitamin D intake and the $25(\mathrm{OH}) \mathrm{D}$ concentration among the participants were low. The correlation coefficients were comparable with our study, being the highest between FFQ and recall ( $r$ 0.69) and the lowest between biomarker and recall ( $r 0 \cdot 40)$. However, in the Serbian study, blood samples were available only from twenty participants. Studies from Canada showed good correlations between a selfadministered vitamin D FFQ and S-25(OH)D ( $r 0.48)$ as well as 7-d FR $(r 0.53)^{(7)}$ and between an interview-administered vitamin $\mathrm{D}$ and Ca FFQ and 5-d FR ( $r 0.63$ and $r 0.89$, respectively $)^{(8)}$. In a Korean study, consumption of vitamin D-rich products based on the FFQ was related to S-25(OH)D status ${ }^{(9)}$. In all these abovementioned studies, supplement use was taken into account ${ }^{(7-10)}$. We found in the present study that vitamin D intake from supplements is easier to collect with greater accuracy than the intake from dietary sources. When supplementary intake is high, it reflects more on the total intake, attenuating the impacts of dietary intake on the biomarker - S-25(OH)D. This was seen as better correlations with higher supplement intake levels. In our study, for $\mathrm{Ca}$, no comparison with a biomarker was made because no reliable biomarker for Ca status exists ${ }^{(18)}$.

In Finland, several studies have validated FFQs that collect, among other nutrients, data on vitamin D and Ca intakes, but 
none of the studies have focused on only these two nutrients. The correlations between self-administered FFQ and FR (3-14d) were 0.19-0.44 for vitamin D and 0.40-0.63 for $\mathrm{Ca}^{(25-29)}$. Three of these studies also assessed the reproducibility of the FFQ within a 3-month interval, with correlations ranging from 0.58 to 0.77 for vitamin $D$ and from 0.64 to 0.72 for $\mathrm{Ca}^{(25-27)}$. In our study, the correlations between the two methods were 0.75 for vitamin $\mathrm{D}$ and 0.70 for $\mathrm{Ca}$, and those in the reproducibility study were 0.70 for vitamin $\mathrm{D}$ and 0.76 for $\mathrm{Ca}$. This may reflect more accurate estimations of intake by an interview-driven FFQ. Nevertheless, in the review of Henríquez-Sánchez et al. ${ }^{(6)}$ on FFQ validation studies for vitamin $\mathrm{D}$ intake, the correlations ranged from 0.51 between FFQ and FR to 0.61 between FFQ and dietary recalls, thus being quite similar to the Finnish studies. The weighted $\kappa$ analysis in our study showed almost moderate agreement for the reproducibility of the FFQ. Further, in the cross-classification analysis, no major misclassification was present.

Our questions on frequency and portion sizes were closed, which is recommended ${ }^{(5)}$. The FFQ was administered before the FR, as suggested. Moreover, the reference period of 8 weeks is considered to be long enough to assess reproducibility of the questionnaire $^{(19)}$. Cade et al. ${ }^{(5)}$ state that age, ethnic group, sex and health status can have an effect on the results of a validation study. Our age group was quite narrow and participants were all healthy Caucasians of Finnish origin of the same sex (females). The participants were mainly recruited in the University Campus area, and the results for other socioeconomic groups might have been different because better vitamin $\mathrm{D}$ status has been shown to be associated with higher socio-economic class ${ }^{(30)}$.

Vitamin D correlates only weakly with energy intake; thus, there was no need for energy adjustment ${ }^{(19)}$. Ca usually correlates with energy intake. However, the FFQ was developed to assess only intake of these two nutrients, and the energy intake was not available. Moreover, we were especially interested in the absolute intake of these two nutrients. It is important to note that the 3-d FR used in our validation study may have underestimated the vitamin $\mathrm{D}$ intake due to the short recording period. Vitamin D-rich fish sources may not have been comprehensively included in those $3 \mathrm{~d}$. The FFQ was interviewdriven, which can be considered a limitation if aiming to develop a very low-cost assessment method. In Finland, where the food chain contains a wide variety of vitamin D-fortified products with differing amounts of the vitamin, it could be challenging to complete a self-administered FFQ, even with the aid of a picture booklet. The supplement use was clarified by some separate questions, which was less burdensome for the participants to report than the dietary intake (i.e. food intake). Supplementary vitamin D intake comprises a greater amount of total vitamin D intake than in the past in Finland ${ }^{(4)}$, which was also observed in the present study. This proves a need to validate the FFQ with different supplementation levels, at least when validating against a biomarker.

The biomarker chosen was S-25(OH)D, which is considered an unbiased marker of vitamin D status. It reflects both sunlight and dietary and supplementary vitamin D intake. During the Finnish winter seasons, not enough sunlight is available to produce vitamin D in the skin. Thus, dietary and supplementary vitamin $D$ have the main impact on S-25(OH)D status ${ }^{(1)}$, providing the best possible timing for a validity study.

\section{Conclusions}

The FFQ provided a reasonable estimation of vitamin D and Ca intakes in Finnish women as compared with FR and the biomarker for vitamin D status, S-25(OH)D. The results are comparable with earlier validation studies. This is the first FFQ validation study for vitamin D intake using the method of triads, taking into account vitamin D intake from the FFQ, FR and S-25 $(\mathrm{OH}) \mathrm{D}$, conducted in a region with wide food fortification and considerably high supplemental consumption. The methods correlated well, but observed variation in the estimates could have been avoided with a longer food recording period and larger number of participants; thus, the results of the method of triads should be interpreted with caution. Moreover, the Bland-Altman analysis showed wide limits of agreement between FFQ and FR, indicating individual differences in the estimations at different intake levels. The observed differences in the correlations between FFQ and S-25(OH)D among different supplementation levels indicate the importance of using varying methods in the validation studies as well as the significance of being aware of the strong effect of supplementation when validating against a biomarker. For further use, the FFQ should be validated in other population groups. Moreover, if the variety of vitamin D-fortified products on the market increases, the FFQ will need to be updated and re-validated with a recommendation to use a larger sample size.

\section{Acknowledgements}

The authors thank Hannu Rita and Elina Vaara for statistical advice.

This work was undertaken within ODIN WP5 (www.odinvitamin D.eu), which is funded by the European Commission (grant agreement 613977). Other funding sources are the University of Helsinki and the Federation for Finnish Food and Drink Industry. The funders had no role in the design, analysis or writing of this article.

The author contributions are as follows: S. T. I., M. E. and C. J. E. L.-A. designed the study. All the authors participated in the development of the FFQ. E. S., P. S. and E. M. S. collected the data. S. T. I. performed the statistical analyses with help from M. E. S. T. I. drafted the manuscript. All the authors reviewed and approved the final draft of the manuscript.

The authors have no conflicts of interest to declare.

\section{References}

1. Lamberg-Allardt C, Brustad M, Meyer HE, et al. (2013) Vitamin D - a systematic review for the 5 th edition of the Nordic Nutrition Recommendations. Food Nutr Res 57, 10.3402/fnr.v57i0.22671; eCollection 2013.

2. Lamberg-Allardt CJ, Outila TA, Kärkkäinen MU, et al. (2001) Vitamin D deficiency and bone health in healthy adults in Finland: could this be a concern in other parts of Europe? J Bone Miner Res 16, 2066-2073. 
3. Raulio S, Erlund I, Männistö S, et al. (2014) Nutrition policy actions performed in Finland in order to increase the vitamin D intake and serum 25OHD concentration in the population. Abstract in III World Congress of Public Health Nutrition, Las Palmas de Gran Canaria, Spain, 9-12 September 2014.

4. Helldán A, Raulio S, Kosola M, et al. (2013) The National FINDIET 2012 Survey. Reports of Institution of Health and Welfare, 16/2013. Tampere, Finland: Juvenes Print.

5. Cade J, Thompson R, Burley V, et al. (2001) Development, validation and utilization of food-frequency questionnaires. Public Health Nutr 5, 567-587.

6. Henríguez-Sánchez P, Sánchez-Villegas A, Doreste-Alonso J, et al. (2009) Dietary assessment methods for micronutrient intake: a systematic review. Br J Nutr 102, S10-S37.

7. Wu H, Gozdzik A, Barta JL, et al. (2009) The development and evaluation of a food frequency questionnaire used in assessing vitamin D intake in a sample of healthy young Canadian adults of diverse ancestry. Nutr Res 29, 255-261.

8. Pritchard JM, Seechurn T \& Atkinson SA (2010) A food frequency questionnaire for the assessment of calcium, vitamin D and vitamin K: a pilot validation study. Nutrients $\mathbf{2}$ 805-819.

9. Yu A, Kim J, Kwon O, et al. (2013) The association between serum 25-hydroxyvitamin D concentration and consumption frequencies of vitamin D sources in Korean adolescents. Clin Nutr Res 2, 107-114.

10. Djekic-Ivankovic M, Weiler HA, Nikolic M, et al. (2015) Validity of an FFQ assessing the vitamin D intake of young Serbian women living in a region without food fortification: the method of triads model. Public Health Nutr (Epublication ahead of print version 11 May 2015).

11. Välimäki MJ, Tiihonen M, Laitinen K, et al. (1994) Bone mineral density measured by dual-energy X-ray absorptiometry in patients on antiepileptic drugs. J Bone Miner Res 9 , 631-637.

12. Outila TA, Kärkkäinen MUM, Seppänen RH, et al. (2000) Dietary intake of vitamin D in premenopausal, healthy vegans was insufficient to maintain concentrations of serum 25-hydroxyvitamin D and intact parathyroid hormone within normal ranges during the winter in Finland. J Am Diet Assoc 100, 434-441.

13. Sääkșärvi K \& Reinivuo H (2004) Ruokamittoja (Guide for Portion Sizes). Publications of National Public Health Institute B15/2004. Helsinki: National Public Health Institute (in Finnish).

14. Cohen J (1968) Weighted kappa: nominal scale agreement with provision for scaled disagreement or partial credit. Psychol Bull 70, 213-220.

15. Altman DG \& Bland JM (1983) Measurement in medicine: the analysis of method comparison studies. Statistician 32, 307-317.

16. Yokota RTdC, Miyazaki ES \& Ito MK (2010) Applying the triads method in the validation of dietary intake using biomarkers. Cad Saude Publica 26, 2027-2037.
17. Cashman KD, Dowling K, Skrabakova Z, et al. (2015) Standardizing serum 25-hydroxyvitamin D data from four Nordic population samples using the Vitamin D Standardization Program protocols: shedding new light on vitamin D status in Nordic individuals. Scan J Clin Lab Invest 75, 549-561.

18. National Nutrition Council (2014) Suomalaiset ravitsemussuositukset - Terveyttä ruoasta. (Finnish Nutrition Recommendations - Health from Food). Helsinki: Juvenes Print (in Finnish)

19. Willett W (2013) Nutritional Epidemiology, 3rd ed. New York: Oxford University Press.

20. Ocké MC \& Kaaks RJ (1997) Biochemical markers as additional measurements in dietary validity studies: application of the method of triads with examples from the European Prospective Investigation into Cancer and Nutrition. Am J Clin Nutr 65, Suppl. , 1240S-1245S.

21. McNaughton SA, Marks GC, Gaffney P, et al. (2005) Validation of a food frequency questionnaire assessment of carotenoid and vitamin $\mathrm{E}$ intake using weighed food records and plasma biomarkers: the method of triads model. Eur J Clin Nutr 59, 211-218.

22. McNaughton SA, Hughes MC \& Marks GC (2007) Validation of a FFQ to estimate the intake of PUFA using plasma phospholipid fatty acids and weighed food records. Br J Nutr $\mathbf{9 7}$, 561-568.

23. Masson LF, McNeill G, Simpson JA, et al. (2003) Statistical approaches for assessing the relative validity of a foodfrequency questionnaire: use of correlation coefficients and the kappa statistics. Public Health Nutr 6, 313-321.

24. Jacques PF, Sulsky SI, Sadowski JA, et al. (1993) Comparison of micronutrient intake measured by a dietary questionnaire and biochemical indicators of micronutrient status. Am J Clin Nutr 57, 182-189.

25. Pietinen P, Hartman AM, Haapa E, et al. (1988) Reproducibility and validity of dietary assessment instruments I. A selfadministered food use questionnaire with a portion size picture book. Am J Epidemiol 128, 655-666.

26. Männistö S, Virtanen M, Mikkonen T, et al. (1996) Reproducibility and validity of a food frequency questionnaire in a case-control study on breast cancer. J Clin Epidemiol 49, 401-409.

27. Erkkola M, Karppinen M, Javanainen J, et al. (2001) Validity and reproducibility of a food frequency questionnaire for pregnant Finnish women. Am J Epidemiol 154, 466-476.

28. Paalanen L, Männistö S, Virtanen MJ, et al. (2006) Validity of a food frequency questionnaire varied by age and body mass index. J Clin Epidemiol 59, 994-1001.

29. Erkkilä AT, Järvinen R, Karvonen H, et al. (2011) Validation of a semi-quantitative FFQ using food records as a reference in older women in the Kuopio Fracture Prevention Study (OSTPRE-FPS). Public Health Nutr 15, 635-639.

30. Jääskeläinen T, Knekt P, Marniemi J, et al. (2013) Vitamin D status is associated with sociodemographic factors, lifestyle and metabolic health. Eur J Nutr 52, 513-525. 